\title{
ON THE GEOMETRY OF MULTISYMPLECTIC MANIFOLDS
}

\author{
F. CANTRIJN*, A. IBORT and M. DE LEÓN
}

(Received 23 December 1998)

Communicated by R. B. Bartnik

\begin{abstract}
A multisymplectic structure on a manifold is defined by a closed differential form with zero characteristic distribution. Starting from the linear case, some of the basic properties of multisymplectic structures are described. Various examples of multisymplectic manifolds are considered, and special attention is paid to the canonical multisymplectic structure living on a bundle of exterior $k$-forms on a manifold. For a class of multisymplectic manifolds admitting a 'Lagrangian' fibration, a general structure theorem is given which, in particular, leads to a classification of these manifolds in terms of a prescribed family of cohomology classes.
\end{abstract}

1991 Mathematics subject classification (Amer. Math. Soc.): primary 53C15, 58Axx.

\section{Introduction}

A canonical structure which naturally appears in the covariant Hamiltonian formulation of classical field theories, is that of a closed (more precisely, exact) nondegenerate differential form of degree $k+1$ on a bundle of exterior $k$-forms over a (fibred) manifold (see $[6,10-12])$. Nondegenerate here means that the characteristic distribution of the form consists of the zero vector field only. This form yields a natural extension of the canonical symplectic form on a cotangent bundle, and we will follow the previous authors in calling it a multisymplectic form. Multisymplectic structures in field theory play a role similar to that of symplectic structures in classical mechanics. In particular, they provide a natural geometric framework adapted to the variational character of the theory.

*Senior Research Associate at the Fund for Scientific Research - Flanders (Belgium).

(C) 1999 Australian Mathematical Society 0263-6115/99 \$A2.00+0.00 
The use of multisymplectic structures in field theory seems to originate from Tulczyjew's 1968 Warsaw seminars on 'Phase Space Theory' (see also [4] for some historical comments). Many of the ideas which were presented in these seminars have later gradually found their way into the literature (see $[19,30,33])$. In the seventies, Kijowski and Szczyrba have developed a finite dimensional canonical formalism for classical field theory which explicitly relies on the notions of multiphase space and multisymplectic structure, first introduced by Tulczyjew (see $[17,18]$ ). Around the same time, similar ideas were also developed by others (see $[8,9]$ ).

In the present paper we extend the concept of multisymplectic form to any closed and nondegenerate differential form on a manifold. Symplectic forms and volume forms in particular belong to this category. Apart from these two extreme cases there are, however, various other interesting types of manifolds which carry a distinguished multisymplectic form such as, for instance, quaternionic (almost) Kähler manifolds. The study of these manifolds may therefore benefit from a better insight into the geometry of multisymplectic structures. Recently, one of us has also pointed out the existence of multisymplectic structures on the moduli spaces of some YangMills theories in dimensions 3 and 4 [15]. To our knowledge, the first detailed study devoted to the structure of multisymplectic manifolds, is due to Martin [22]. Although Martin's definition of a multisymplectic form is somewhat more restrictive than ours, and appears to be less suited for applications to canonical field theory, his work has certainly shed some light on the nice geometry induced by such forms and he has also indicated some other potential areas of application (see also [23]). All of this has motivated us to initiate a systematic study of general multisymplectic structures. Attention will thereby be paid to purely theoretical aspects as well as to applications. In the present paper we will confine ourselves to some of the basic concepts and properties related to the existence of a multisymplectic structure on a manifold.

The structure of the paper is as follows. In Section 2, Section 3 and Section 4, we will deal with the linear theory, that is the study of multisymplectic vector spaces. In Section 2, after some introductory definitions, we briefly comment on the general classification problem of linear multisymplectic structures, and certain canonical models of multisymplectic vector spaces are described. Section 3 starts with a discussion of the notion of orthogonal complement of a subspace of a multisymplectic vector space. It is pointed out that for a multisymplectic form of degree $k+1>2$ there is a possibility of defining $k$ different types of 'orthogonal complement', each giving rise to a corresponding notion of isotropic, coisotropic and Lagrangian subspace. In Section 4, necessary and sufficient conditions are established for a multisymplectic vector space to be isomorphic to a canonical one. Section 5 then passes to the study of multisymplectic manifolds. The concepts introduced in the previous sections are extended to the differentiable setting, and some interesting examples of multisymplectic manifolds are given. The canonical multisymplectic structure on a bundle of exterior 
forms is studied in Section 6. Finally, in Section 7 we introduce a notion of polarized multisymplectic manifold and present a general structure theorem for such manifolds. Throughout this paper, all manifolds and related objects (maps, vector fields, forms, ... ) are assumed to be of class $C^{\infty}$.

To close this introduction we wish to emphasize that our notion of multisymplectic structure should be clearly distinguished from certain other structures which have recently been discussed in the literature such as, for instance, $k$-almost cotangent structures [20]. The latter, in particular, constitute a generalization of the so-called polysymplectic structures [13] and $k$-symplectic structures [2,3]. A polysymplectic structure refers to a particular vector valued differential form on (the affine dual of) a first order jet bundle, which plays a key role in some geometric approaches to Hamiltonian field theory (see $[13,16,28,29])$. A $k$-symplectic structure on a manifold is determined by $k$ closed 2 -forms which verify some compatibility conditions. A canonical model of a $k$-symplectic structure is provided by the 'generalized symplectic structure', determined by the soldering form on the linear frame bundle of a manifold, which has recently been studied by Norris et al. $[7,26]$. In an appropriate setting it can be shown that polysymplectic and $k$-symplectic structures are essentially equivalent and can be identified with an integrable $k$-almost cotangent structure.

\section{Multisymplectic vector spaces}

Given a vector space $V$ we will denote the space of exterior $k$-forms on $V$ by $\bigwedge^{k} V^{*}$. By an exterior form (or simply a form) on a vector space, we mean an alternating multilinear function on that space with values in the field of scalars. In particular, we have $\bigwedge^{l} V^{*}=V^{*}$, that is the dual space of $V$. The contraction of a vector $v \in V$ and an exterior $k$-form $\omega$ on $V$ will be denoted by $i_{v} \omega$.

DEFINITION 2.1. (i) A multisymplectic vector space $(\mathscr{V}, \omega)$ of order $k+1$ consists of a vector space $\mathscr{V}$ and an exterior $(k+1)$-form $\omega$ on $\mathscr{V}$ (with $1<k+1 \leq \operatorname{dim} \mathscr{V}$ ) which is nondegenerate in the sense that $i_{v} \omega=0$ if and only if $v=0$. The form $\omega$ is called a multisymplectic form (of degree $k+1$ ).

(ii) Two multisymplectic vector spaces $(\mathscr{V}, \omega)$ and $(\tilde{\mathscr{V}}, \tilde{\omega})$ of equal order $k+1$ are said to be isomorphic if there exists a linear isomorphism $\Psi: \mathscr{V} \mapsto \tilde{\mathscr{V}}$ such that

$$
\tilde{\omega}\left(\Psi\left(v_{1}\right), \ldots, \Psi\left(v_{k+1}\right)\right)=\omega\left(v_{1}, \ldots, v_{k+1}\right)
$$

for all $v_{i} \in \mathscr{V}(i=1, \ldots, k+1)$.

We note that for $k=1$ the above definition reduces to that of a symplectic vector space. Nondegeneracy of a multisymplectic form $\omega$ of degree $k+1$ means that the 
induced linear map

$$
\hat{\omega}: \mathscr{V} \rightarrow \bigwedge^{k} \mathscr{V}^{*}, \quad v \mapsto i_{v} \omega
$$

is injective. Alternatively, one can say that an exterior form $\omega$ on a vector space $\mathscr{V}$ is multisymplectic if its rank is equal to the dimension of $\mathscr{V}$. It immediately follows that a $n$-dimensional vector space cannot admit a multisymplectic form of degree $n-1$. Indeed, it is known that a (nonzero) ( $n-1)$-form on a $n$-dimensional space can only have rank $n-1$. (In fact, it can be shown more generally that a $p$-form can never have rank $p+1)$. In the sequel we will restrict ourselves to the case of real finite dimensional vector spaces. Much of what follows can be easily carried over, however, to the case of complex and infinite dimensional linear spaces.

EXAMPLES. 1. A volume form on a $n$-dimensional space defines a multisymplectic structure of order $n$.

2. Consider a 6-dimensional space $\mathscr{V}_{0}$ with basis $\left\{e_{i} \mid i=1, \ldots, 6\right\}$ and dual basis $\left\{\theta^{i} \mid i=1, \ldots, 6\right\}$. One easily verifies that the 3 -form

$$
\omega_{0}=\theta^{1} \wedge \theta^{4} \wedge \theta^{5}+\theta^{2} \wedge \theta^{4} \wedge \theta^{6}+\theta^{3} \wedge \theta^{5} \wedge \theta^{6}+\theta^{4} \wedge \theta^{5} \wedge \theta^{6}
$$

is nondegenerate in the sense specified above and, hence, induces a multisymplectic structure of order 3 on $\mathscr{V}_{0}$. We will return to this example later on.

Vector spaces equipped with an exterior form of a given rank have already been considered extensively in the literature. For completeness we recall here some interesting results from a paper by Martinet [24]. Let $\Sigma_{n, r}^{k+1}$ denote the set of exterior $(k+1)$-forms of rank $r$ on a $n$-dimensional vector space $\mathscr{V}$. Martinet has demonstrated that each non-void $\Sigma_{n, r}^{k+1}$ is a regular submanifold of $\bigwedge^{k+1} \mathscr{V}^{*}$ of dimension $C_{r}^{k+1}+r(n-r)$ (or 0 for $r=0$ ), and that these submanifolds determine a stratification of $\bigwedge^{k+1} \mathscr{V}^{*}$. The closure of $\Sigma_{n, r}^{k+1}$, which is given by $\overline{\Sigma_{n, r}^{k+1}}=\bigcap_{s \leq r} \Sigma_{n, s}^{k+1}$, is an algebraic manifold. Furthermore, it can be shown that for $3 \leq k+1 \leq n-3$ and for each allowable value of $r$ (that is $r=0, k+1, k+3, \ldots, n), \Sigma_{n, r}^{k+1}$ is non-void and, in particular, for $r=n$ it yields an open dense submanifold of $\bigwedge^{k+1} \mathscr{V}^{*}$. Hence, on vector spaces of dimension $n \geq 6$, multisymplectic structures of order $3 \leq k+1 \leq n-3$ are generic.

Returning to the case of multisymplectic forms $(r=n)$, an important problem concerns their classification. Classifying multisymplectic structures amounts to describe the orbits of the group of linear automorphisms $G l(\mathscr{V})$ of $\mathscr{V}$ acting on $\Sigma_{n, n}^{k+1}$ (see $[24,27,35,36])$. For the symplectic case, with $n$ even and $k+1=2$, the situation is well-known: $G l(\mathscr{V})$ acts transitively on $\Sigma_{n, n}^{2}$. For a discussion of the case $k+1=n-2$, we may again refer to the paper by Martinet. The classification of multisymplectic structures of order 3 on real vector spaces up to dimension 7 has also been 
established (see $[27,36]$ and references therein). In particular, on a 6-dimensional space there are 2 , and on a 7 -dimensional space there are 8 equivalence classes (that is $G l(\mathscr{V})$-orbits) of multisymplectic forms of degree 3. To our knowledge, apart from these particular cases, the general classification problem of multisymplectic structures largely remains open up to date. To illustrate the complexity of the problem, we may still point out that for $n \geq 9$ and $3 \leq k+1 \leq n-3$, it is easy to show that the dimension of $\Sigma_{n, r}^{k+1}$ is greater than the dimension of $G l(\mathscr{V})$. The orbit space $\Sigma_{n, r}^{k+1} / G l(\mathscr{V})$ turns out to be a manifold of dimension greater than 1, the description of which, however, is not yet known.

In what follows we will concentrate on a particular class of multisymplectic structures that possess some remarkable properties which, in a sense to be discussed below, extend the properties of symplectic structures.

It is well-known that for any vector space $V$, the space $V \times V^{*}$ admits a canonical symplectic form $\Omega$, defined by

$$
\Omega\left(\left(v_{1}, \alpha_{1}\right),\left(v_{2}, \alpha_{2}\right)\right)=\alpha_{2}\left(v_{1}\right)-\alpha_{1}\left(v_{2}\right)
$$

for $v_{1}, v_{2} \in V$ and $\alpha_{1}, \alpha_{2} \in V^{*}$ (see [1,21]). This structure has the following natural extension to the multisymplectic setting. For any $k$, with $1 \leq k \leq \operatorname{dim} V$, the space $V \times \wedge^{k} V^{*}$ can be equipped with a canonical exterior $(k+1)$-form $\Omega$ which is given by

$$
\Omega\left(\left(v_{1}, \alpha_{1}\right), \ldots,\left(v_{k+1}, \alpha_{k+1}\right)\right)=\sum_{i=1}^{k+1}(-1)^{i} \alpha_{i}\left(v_{1}, \ldots, \hat{v}_{i}, \ldots, v_{k+1}\right)
$$

for $v_{i} \in V$ and $\alpha_{i} \in \bigwedge^{k} V^{*}$, where $\hat{v}_{i}$ means that $v_{i}$ is omitted. It is now a simple exercise to show that the following holds.

PROPOSITION 2.2. $\left(V \times \wedge^{k} V^{*}, \Omega\right)$ is a multisymplectic vector space of order $k+1$.

REMARKS. 1. The (irreducible) length of an exterior form $\omega$ can be defined as the number of decomposable forms appearing in the shortest representation of $\omega$ as a sum of decomposable forms. It has been pointed out in [24] that both the rank and the length of an exterior form are invariants under the group of linear automorphisms. One can now add to the statement of the previous proposition that the multisymplectic form $\Omega$ has length $C_{n}^{k}$, where $n=\operatorname{dim} V$. (A proof of this can be easily deduced from the discussion in Section 4.)

2. If $k=\operatorname{dim} V, \bigwedge^{k} V^{*}$ is a 1 -dimensional linear space. Fixing a volume form $\bar{v}$ on $V$, each exterior $k$-form is a constant multiple of $\bar{\nu}$. The canonical multisymplectic form $\Omega$ then yields a volume form on $V \times \wedge^{k} V^{*}$, and putting $\alpha_{i}=\lambda_{i} \bar{\nu}$ with $\lambda_{i} \in \mathbb{R}$, (3) reads

$$
\Omega\left(\left(v_{1}, \alpha_{1}\right), \ldots,\left(v_{k+1}, \alpha_{k+1}\right)\right)=\sum_{i=1}^{k+1}(-1)^{i} \lambda_{i} \bar{\nu}\left(v_{1}, \ldots, \hat{v}_{i}, \ldots, v_{k+1}\right) .
$$


For convenience, we introduce the shorthand notation

$$
\mathscr{V}_{V}^{k}=V \times \wedge^{k} V^{*}
$$

We will now identify an important class of multisymplectic subspaces of $\left(\mathscr{V}_{V}^{k}, \Omega\right)$. For the remainder of this section, both $V$ and $k$ (with $1 \leq k \leq \operatorname{dim} V$ ) are supposed to be fixed.

Let $\pi: V \longrightarrow W$ be a linear surjective map of $V$ onto a vector space $W$ and consider the short exact sequence

$$
0 \rightarrow \operatorname{ker} \pi \hookrightarrow V \stackrel{\pi}{\rightarrow} W \rightarrow 0 .
$$

For any $r$, with $0 \leq r \leq k-1$, we denote by $\bigwedge_{r}^{k} \pi$ the space of exterior $k$-forms on $V$ which vanish whenever $r+1$ of its arguments belong to ker $\pi$ (that is, are vertical with respect to the 'fibration' $\pi$ ). More explicitly, a $k$-form $\alpha$ on $V$ belongs to $\bigwedge_{r}^{k} \pi$ if and only if

$$
i_{v_{1} \wedge \cdots \wedge v_{r+1}} \alpha=0,
$$

for all $v_{1}, \ldots, v_{r+1} \in \operatorname{ker} \pi$. Elements of $\bigwedge_{r}^{k} \pi$ are also called $(k-r)$-horizontal $k$-forms on $V$ (with respect to $\pi$ ) and we have the following filtration:

$$
\bigwedge_{0}^{k} \pi \subseteq \bigwedge_{1}^{k} \pi \subseteq \cdots \subseteq \bigwedge_{k-1}^{k} \pi \subseteq \bigwedge^{k} V^{*}
$$

In particular, note that $\bigwedge_{0}^{k} \pi \cong \bigwedge^{k} W^{*}$, and if $\operatorname{dim}(\operatorname{ker} \pi)<k$, then $\bigwedge_{r}^{k} \pi=\bigwedge^{k} V^{*}$ for all $r \geq \operatorname{dim}(\operatorname{ker} \pi)$. Next, put

$$
\mathscr{V}_{\pi}^{(k, r)}=V \times \bigwedge_{r}^{k} \pi .
$$

Clearly, for each $0 \leq r<k, \mathscr{V}_{\pi}^{(k, r)}$ is a subspace of $\mathscr{V}_{V}^{k}$. Now, whenever $k-r \leq \operatorname{dim} W$, one easily verifies (using a similar argument as in the proof of Proposition 2.2) that the restriction of the canonical multisymplectic form $\Omega$ to $\mathscr{V}_{\pi}^{(k, r)}$ is also nondegenerate and, hence, induces a multisymplectic structure on $\mathscr{V}_{\pi}^{(k, r)}$. This induced multisymplectic form is still given by the expression (3), with $v_{i} \in V$ and $\alpha_{i} \in \bigwedge_{r}^{k} \pi$ and, therefore, we will also denote it by $\Omega$. We thus have the following result.

PROPOSITION 2.3. For each $r$, with $0 \leq r \leq k-1$ and $k-r \leq \operatorname{dim} W,\left(\mathscr{V}_{\pi}^{(k, r)}, \Omega\right)$ is a multisymplectic vector space of order $k+1$.

This proposition tells us that any fibration $\pi$ of a given vector space $V$, gives rise to a family of multisymplectic vector subspaces of $\left(\mathscr{V}_{V}^{k}, \Omega\right)$. In particular, the multisymplectic structure obtained by restricting $\Omega$ to $\mathscr{V}_{\pi}^{(k, 1)}=V \times \bigwedge_{1}^{k} \pi$ is a linear model for the canonical multisymplectic structure arising in the covariant Hamiltonian formulation of field theory (see $[6,10,12])$. 


\section{Isotropic, coisotropic and Lagrangian subspaces}

On a symplectic vector space there is a natural notion of orthogonal complement of a subspace with respect to the given symplectic 2-form. On a multisymplectic space of order $k+1>2$, however, there are several options for defining some kind of 'orthogonality'. Indeed, let $(\mathscr{V}, \omega)$ be an arbitrary multisymplectic vector space with $\omega$ a nondegenerate $(k+1)$-form, and let $W$ be a subspace of $\mathscr{V}$. For each $l$, with $1 \leq l \leq k$, the $l$-th orthogonal complement of $W$ is the linear subspace of $\mathscr{V}$ defined by

$$
W^{\perp, l}=\left\{v \in \mathscr{V} \mid i_{v \wedge w_{1} \wedge \cdots \wedge w_{l}} \omega=0, \text { for all } w_{i} \in W, i=1, \ldots, l\right\} .
$$

From this definition it can immediately be deduced that for each subspace $W$ there exists a filtration of orthogonal complements

$$
W^{\perp, 1} \subseteq W^{\perp, 2} \subseteq \cdots \subseteq W^{\perp, k}
$$

and, moreover, $W^{\perp, l}=\mathscr{V}$ whenever $l>\operatorname{dim} W$. One also readily verifies that

$$
W \cap W^{\perp, k}=\operatorname{ker}\left(\left.\omega\right|_{W}\right),
$$

where $\left.\omega\right|_{W}$ denotes the restriction of $\omega$ to $W$ and $\operatorname{ker}\left(\left.\omega\right|_{W}\right)=\left\{w \in W \mid i_{w}\left(\left.\omega\right|_{W}\right)=0\right\}$.

Although it will soon turn out that only the orthogonal complements of 'lowest' and 'highest' order (that is for $l=1$ and $l=k$, respectively) have a significant role to play in the further development of the theory, we will nevertheless, for the sake of completeness, also present some properties of general orthogonal complements.

PROPOSITION 3.1. Let $(\mathscr{V}, \omega)$ be a multisymplectic vector space of order $k+1$, and let $U$ and $W$ be arbitrary subspaces of $\mathscr{V}$. Then, for any $l, l_{1}, l_{2} \in\{1, \ldots, k\}$ :

(i) $\{0\}^{\perp, l}=\mathscr{V}$ and $\mathscr{V}^{\perp, l}=\{0\}$;

(ii) $U \subset W \Longrightarrow W^{\perp . l} \subset U^{\perp, l}$;

(iii) $(U+W)^{\perp, l} \subset U^{\perp, l} \cap W^{\perp, l}$;

(iv) $U^{\perp, l_{1}} \cap W^{\perp, l_{2}} \subset(U+W)^{\perp, l_{1}+l_{2}-1}$ for $l_{1}+l_{2} \leq k+1$;

(v) $U^{\perp, l_{1}}+W^{\perp, l_{2}} \subset(U \cap W)^{\perp, \bar{l}}$ with $\bar{l}=\max \left\{l_{1}, l_{2}\right\}$.

Proof. The proofs of (i) and (ii) follow immediately from the definition of $l$-th orthogonal complement (and the nondegeneracy of $\omega$ ).

(iii) Since $U \subset U+W$ and $W \subset U+W$, (iii) is an immediate consequence of (ii). (iv) Let $v \in U^{\perp, l_{1}} \cap W^{\perp, l_{2}}$, with $l_{1}+l_{2} \leq k+1$. Take $l_{1}+l_{2}-1$ arbitrary elements of $U+$ $W: u_{1}+w_{1}, \ldots, u_{l_{1}+l_{2}-1}+w_{l_{1}+l_{2}-1}$. Then, $\omega\left(v, u_{1}+w_{1}, \ldots, u_{l_{1}+l_{2}-1}+w_{l_{1}+l_{2}-1}, \ldots\right)$ can be expanded into a sum of terms containing $p$ elements $u_{i} \in U$ and $q$ elements 
$w_{i} \in W$, with $p+q=l_{1}+l_{2}-1$. If $p<l_{1}$, then $q \geq l_{2}$, and the corresponding term in the expansion vanishes because $v \in W^{\perp, l_{2}}$. On the other hand, the terms for which $p \geq l_{1}$ vanish since $v \in U^{1, l_{1}}$. Therefore, $\omega\left(v, u_{1}+w_{1}, \ldots, u_{l_{1}+l_{2}-1}+w_{l_{1}+l_{2}-1}, \ldots\right)=$ 0 for all $u_{i}+w_{i} \in U+W$ and so $v \in(U+W)^{\perp, l_{1}+l_{2}-1}$.

(v) Since $U \cap W \subset U$, combination of (ii) and (4) yields $U^{\perp, l_{1}} \subset(U \cap W)^{\perp, \bar{l}}$. Similarly, from $U \cap W \subset W$ we derive that $W^{1, l_{2}} \subset(U \cap W)^{\perp, \bar{l}}$ and the result now readily follows.

In the symplectic case $(k+1=2)$, the above relations reduce to the well-known properties of the orthogonal complement of a subspace of a symplectic vector space, see [21]. Moreover, when putting $l=l_{1}=l_{2}=1$ in (iii) and (iv) of the previous proposition, we immediately derive the following result.

COROLlARY 3.2. For any two subspaces $U$ and $W$ of a multisymplectic vector space $(\mathscr{V}, \omega)$

$$
(U+W)^{\perp, 1}=U^{\perp, 1} \cap W^{\perp, 1} .
$$

We can now introduce the following special types of subspaces of a multisymplectic vector space, generalizing the corresponding notions from symplectic geometry.

DEFINITION 3.3. A subspace $W$ of a multisymplectic vector space $(\mathscr{V}, \omega)$ of order $k+1$ is called: $l$-isotropic if $W \subset W^{\perp, l} ;$ l-coisotropic if $W^{\perp, l} \subset W$ and $l$-Lagrangian if $W=W^{\perp, l}$, for $1 \leq l \leq k$. W is a multisymplectic subspace of $(\mathscr{V}, \omega)$ if $W \cap W^{1, k}=\{0\}$.

EXAMPLE. Consider the multisymplectic space $\left(\mathscr{V}_{0}, \omega_{0}\right)$ of order 3 , introduced in Example 2 of the previous section, with $\operatorname{dim} \mathscr{V}_{0}=6$ and $\omega_{0}$ given by (1). Consider the subspaces $W=\operatorname{span}\left\{e_{1}, e_{2}, e_{3}\right\}$ and $U=\operatorname{span}\left\{e_{4}, e_{5}, e_{6}\right\}$. It is straightforward to check that $W=W^{\perp, 1}$ and $U^{\perp, 2}=\{0\}$. Therefore, $W$ is a 1-Lagrangian subspace and $U$ is a multisymplectic subspace of $\mathscr{V}_{0}$.

In view of (4) it is clear that an $l$-isotropic (respectively $l$-coisotropic) subspace is also $l^{\prime}$-isotropic (respectively $l^{\prime \prime}$-coisotropic) for all $l^{\prime}>l$ (respectively for all $l^{\prime \prime}<l$ ). Note also that every subspace $W$ is $l$-isotropic for all $l \geq \operatorname{dim} W$. Moreover, one easily verifies, taking into account (ii) of Proposition 3.1, that every subspace (of a multisymplectic space) containing a $l$-coisotropic subspace is itself $l$-coisotropic, and that every subspace of a $l$-isotropic subspace is $l$-isotropic.

In the next proposition we collect a few other interesting properties concerning the concepts of isotropic, coisotropic and Lagrangian subspaces.

PROPOSITION 3.4. Let $(\mathscr{V}, \omega)$ be a multisymplectic vector space, with $\operatorname{dim} \mathscr{V}=n$ and $\omega$ of degree $k+1 \leq n$. Then: 
(i) Each subspace of dimension 1 (respectively codimension 1) is 1-isotropic (respectively $k$-coisotropic).

(ii) If $U$ is a 1-isotropic subspace of $\mathscr{V}$, then codim $U \geq k$.

(iii) If $U$ is a l-isotropic subspace of $\mathscr{V}$, then for every $l^{\prime} \geq l$ there exists a $l^{\prime}$-Lagrangian subspace which contains $U$.

(iv) If $k+1=n$ (that is $\omega$ is a volume form on $\mathscr{V}$ ), then every subspace $U$ of $\mathscr{V}$ is $l$-Lagrangian, with $l=\operatorname{dim} U$ and, moreover, $U^{\perp, l^{\prime}}=\{0\}$ for all $l^{\prime}<l$.

PROOF. (i) If $U$ is a 1-dimensional subspace of $\mathscr{V}$ it readily follows from the definition of the orthogonal complement that $U \subset U^{\perp, 1}$, that is $U$ is 1-isotropic.

Let $W$ be a $(n-1)$-dimensional subspace of $\mathscr{V}$ and let $\left\{e_{1}, \ldots, e_{n-1}\right\}$ denote a basis of $W$. For any $v \in W^{\perp, k}$ we then have, by definition,

$$
\omega\left(v, e_{i_{1}}, \ldots, e_{i_{k}}\right)=0
$$

for all $1 \leq i_{1}<\cdots<i_{k} \leq n-1$. If $v \notin W$, then it readily follows that $i_{v} \omega=0$, which contradicts the assumed nondegeneracy of $\omega$. Hence, we necessarily have that $W^{\perp, k} \subset W$, that is $W$ is $k$-coisotropic.

(ii) Let $U$ be 1 -isotropic and consider a complementary subspace $W$ in $\mathscr{V}$ such that $\mathscr{V}=U \oplus W$. Take any $u \in U^{\perp, 1}$, with $u \neq 0$. By assumption, the $k$-form $i_{u} \omega$ vanishes whenever one of its arguments belongs to $U$. Therefore, the restriction of this form to $W$ should be different from zero, for otherwise $u$ would be a nonzero vector in the kernel of $\omega$. Consequently, we necessarily have $\operatorname{dim} W \geq k$.

(iii) It suffices to prove that every $l$-isotropic subspace $U$ is contained in a $l$ Lagrangian subspace of $\mathscr{V}$ since, as noticed above, a $l$-isotropic subspace is also $l^{\prime}$-isotropic for every $l^{\prime} \geq l$. By assumption, $U \subset U^{\perp, l}$. If $U \neq U^{\perp, l}$, take a vector $v_{1} \in U^{\perp, l} \backslash U$ and put $U_{1}=U \oplus\left\{\lambda v_{1} \mid \lambda \in \mathbb{R}\right\}$. Take $l+1$ arbitrary vectors in $U_{1}$ : $w_{i}=u_{i}+\lambda_{i} v_{1}$, with $u_{i} \in U$ and $\lambda_{i} \in \mathbb{R}$. Using the fact that $U$ is $l$-isotropic and $v_{1} \in U^{\perp, l}$, it follows that

$$
i_{w_{1} \wedge \ldots \wedge w_{l+1}} \omega=0
$$

and, hence, $U_{1} \subseteq U_{1}^{1, l}$, that is $U_{1}$ is also $l$-isotropic. Moreover, from (ii) of Proposition 3.1 it follows that $U_{1}^{\perp, l} \subset U^{\perp, l}$. Summarizing, we thus have the inclusions $U \subset U_{1} \subseteq$ $U_{1}^{\perp, l} \subset U^{\perp, l}$. Continuing this way, we may construct a chain of $l$-isotropic subspaces $U \subset U_{1} \subset U_{2} \ldots$ which necessarily possesses a maximal element $\bar{U}$ for which $\bar{U}=\bar{U}^{\perp, l}$, that is $\bar{U}$ is $l$-Lagrangian.

(iv) Let $\left\{e_{1}, \ldots, e_{\ell}\right\}$ denote a basis of $U$ and complete this to a basis $\left\{e_{1}, \ldots\right.$, $\left.e_{l}, e_{l+1}, \ldots, e_{n}\right\}$ of $\mathscr{V}$. From the definition of orthogonal complement it readily follows that $U \subset U^{\perp . l}$. In order to prove the converse inclusion, take any $v \in U^{\perp, l}$ and put $v=\sum_{i=1}^{n} \lambda_{i} e_{i}$ with $\lambda_{i}$ some real constants. Then, by definition of $U^{\perp, l}$ we 
have

$$
\omega\left(\lambda_{1} e_{1}+\cdots+\lambda_{n} e_{n}, e_{1}, \ldots, e_{l}, e_{l+2}, \ldots, e_{n}\right)=0,
$$

from which we deduce $\lambda_{l+1}=0$. Similarly, replacing $e_{l+2}$ in the previous relation by $e_{l+1}$, we obtain $\lambda_{l+2}=0$, and so on. Eventually, we find that $\lambda_{l+1}=\cdots=\lambda_{n}=0$ and thus $v \in U$. Consequently, $U=U^{\perp . l}$.

For the second part of (iv), take any $v \in U^{\perp, l-1}$ and put again $v=\sum_{i=1}^{n} \lambda_{i} e_{i}$. We now have by definition of $U^{\perp, l-1}$,

$$
\omega\left(v, e_{1}, \ldots, \hat{e}_{i}, \ldots, e_{n}\right)=0
$$

for all $i=1, \ldots, n$, whereby $\hat{e}_{i}$ means that $e_{i}$ is omitted. From this it immediately follows that $\lambda_{i}=0$ for all $i$ and, hence, $v=0$. This proves already that $U^{1, l-1}=\{0\}$ and, in view of (4), it is seen then that the same holds for all $U^{\perp, l^{\prime}}$ with $l^{\prime} \leq l-1$.

Since $\{0\}$ is an $l$-isotropic subspace of $(\mathscr{V}, \omega)$ for all $l$ (compare Proposition 3.1, (i)) we can deduce from (iii) of the previous proposition that there exist $l$-Lagrangian subspaces. In fact, $l$-Lagrangian subspaces are the maximal elements of the (nonempty) partially ordered set of $l$-isotropic subspaces (with respect to the inclusion relation). This argument may also serve as a proof of the existence of Lagrangian subspaces in the infinite dimensional case.

Finally, it should be pointed out that in a multisymplectic space of order $k+1>2$, the $l$-Lagrangian subspaces (for each fixed $l \in\{1, \ldots, k\}$ ) need not all have the same dimension. To illustrate this, we return to our example of the 6-dimensional multisymplectic space $\left(\mathscr{V}_{0}, \omega_{0}\right)$ of order 3 , with basis $\left\{e_{1}, \ldots, e_{6}\right\}$ and with $\omega_{0}$ given by (1). We have seen already above that the 3-dimensional subspace $W=\operatorname{span}\left\{e_{1}, e_{2}, e_{3}\right\}$ of $\mathscr{V}$ is 1-Lagrangian. Now, consider the 2-dimensional subspace $\tilde{W}$ spanned by $e_{2}$ and $e_{5}$. By definition,

$$
\tilde{W}^{\perp, 1}=\left\{v \in \mathscr{V} \mid i_{v} i_{e_{2}} \omega_{0}=i_{v} i_{e_{5}} \omega_{0}=0\right\} .
$$

A simple computation shows that $\tilde{W}^{\perp, 1}=\tilde{W}$ and so $\tilde{W}$ is also 1-Lagrangian. The situation is thus quite different from the (finite dimensional) symplectic case $(k+$ $1=2$ ), where all Lagrangian subspaces have the same dimension, namely half the dimension of the given symplectic space.

\section{Characterization of canonical multisymplectic structures}

Recall from symplectic geometry that, given any (finite dimensional) symplectic vector space $(\mathscr{V}, \omega)$ and an arbitrary Lagrangian subspace $L$ of $\mathscr{V}$ (which always 
exists), one can construct a symplectic isomorphism between $(\mathscr{V}, \omega)$ and $\left(L \times L^{*}, \Omega\right)$, with $\Omega$ given by (2) (see [21]). It follows from this that all symplectic vector spaces of the same dimension 'look the same': they are isomorphic to some $\left(\mathscr{Y}_{L}^{1}, \Omega\right)$. From the discussion of the classification problem of multisymplectic structures (compare Section 2), one can already infer that this will no longer be true in the general multisymplectic case. In particular, the canonical multisymplectic structures $\left(\mathscr{V}_{V}^{k}, \Omega\right)$, introduced in Section 2, cannot be expected to be the natural representatives of all multisymplectic structures of order $k+1$. The main purpose of the present section precisely consists in identifying the class of multisymplectic vector spaces which are isomorphic to one of type $\left(\mathscr{V}_{V}^{k}, \Omega\right)$.

Consider a canonical multisymplectic structure $\left(\mathscr{V}_{V}^{k}, \Omega\right)$, with $\mathscr{V}_{V}^{k}=V \times \wedge^{k} V^{*}$ for some (finite dimensional) vector space $V$ and with $\Omega$ defined by (3). Identifying $V$ and $\wedge^{k} V^{*}$ with the subspaces $V \times\{0\}$ and $\{0\} \times \wedge^{k} V^{*}$ of $\mathscr{V}_{V}^{k}$, respectively, we have the following interesting property.

THEOREM 4.1. $V$ is a k-Lagrangian subspace of $\left(\mathscr{Y}_{V}^{k}, \Omega\right)$ and $\bigwedge^{k} V^{*}$ is a 1-Lagrangian subspace.

PRoOF. By definition, $(v, \alpha) \in \mathscr{V}_{V}^{k}$ belongs to $V^{\perp, k}$ if and only if $\Omega\left((v, \alpha),\left(v_{1}, 0\right)\right.$, $\left.\ldots,\left(v_{k}, 0\right)\right)=0$ for all $v_{i} \in V,(i=1, \ldots, k)$. Using (3), this is still equivalent with

$$
\alpha\left(v_{1}, \ldots, v_{k}\right)=0
$$

for all $v_{i} \in V$, and hence, $\alpha=0$. Consequently, $V^{\perp, k}=V$, that is $V$ is $k$-Lagrangian. Now, let $(v, \alpha)$ be an element of $\left(\bigwedge^{k} V^{*}\right)^{\perp, 1}$. Then,

$$
\Omega\left((v, \alpha),(0, \beta),\left(v_{2}, \alpha_{2}\right), \ldots,\left(v_{k}, \alpha_{k}\right)\right)=0
$$

for all $\beta \in \bigwedge^{k} V^{*}$ and all $\left(v_{i}, \alpha_{i}\right) \in \mathscr{V}_{V}^{k}$ for $i=2, \ldots, k$. Again using (3) we see that $(v, \alpha) \in\left(\bigwedge^{k} V^{*}\right)^{\perp, 1}$ if and only if $i_{v} \beta=0$ for all $\beta \in \bigwedge^{k} V^{*}$, that is if and only if $v=0$. From this we conclude that $\left(\bigwedge^{k} V^{*}\right)^{\perp, 1}=\bigwedge^{k} V^{*}$, which completes the proof of the theorem.

Putting $W=\bigwedge^{k} V^{*}$, again regarded as a subspace of $\mathcal{Y}_{V}^{k}$, we note that

$$
\bigwedge^{k}\left(\mathscr{V}_{V}^{k} / W\right)^{*}=\bigwedge^{k} V^{*}=W
$$

which, in particular, yields

$$
\operatorname{dim} W=\operatorname{dim} \bigwedge^{k}\left(\mathscr{Y}_{v}^{k} / W\right)^{*}
$$


If we put $\operatorname{dim} \mathscr{V}_{v}^{k}=n$ and $\operatorname{dim} W=p$, this dimensional relation reads:

$$
p=\left(\begin{array}{c}
n-p \\
k
\end{array}\right)\left(=C_{n-p}^{k}\right) \text {. }
$$

From this one can already deduce that, given a $n$-dimensional multisymplectic space $(\mathscr{V}, \omega)$ of order $k+1$, a necessary condition for it to be isomorphic to a canonical $n$ dimensional multisymplectic space $\left(\mathscr{V}_{V}^{k}, \Omega\right)$ is that $\mathscr{V}$ admits a 1-Lagrangian subspace $W$ of dimension $p$, such that the relation (6) holds. As a matter of fact, one can prove that this condition is also sufficient. Before doing so, we first need the following useful result which has also been established by Martin (see [23, Proposition 2.4]).

PROPOSITION 4.2. Let $(\mathscr{V}, \omega)$ be a multisymplectic vector space of order $k+1$ and $W$ a 1-Lagrangian subspace such that $\operatorname{dim} W=\operatorname{dim} \bigwedge^{k}(\mathscr{V} / W)^{*}$. Then, there exists a $k$-Lagrangian subspace $V$ which is complementary to $W$, that is such that $\mathscr{V}=V \oplus W$

ProOf. Let $U$ be a $k$-isotropic subspace of $\mathscr{V}$ with $U \cap W=\{0\}$. Such a $U$ always exists and one may even assume $\operatorname{dim} U \geq k$ since, according to (ii) of Proposition 3.4, codim $W \geq k$. First consider the case where $\mathscr{V}=U \oplus W$. One then easily verifies that any vector in $W \cap U^{\perp, k}$ belongs to $\operatorname{ker} \omega$, which necessarily implies that $W \cap U^{\perp . k}=\{0\}$. Since, by assumption, $U \subset U^{\perp, k}$, it follows that $U=U^{\perp, k}$, that is $U$ is $k$-Lagrangian, which already completes the proof in this case.

In case $U \oplus W \neq \mathscr{V}$, one may further proceed along the same lines as in [23, Proposition 2.4]. The idea of the proof is the following. Using a dimensional argument one can deduce that there exists a nonzero vector $u_{1} \in U^{\perp, k}$ which is not contained in $U \cup W$. Putting $U_{1}=U \oplus \operatorname{span}\left\{u_{1}\right\}$, it can be shown that $U_{1}$ is $k$-isotropic. Since, by construction, $U_{1} \cap W=\{0\}$, one can repeat the whole argument starting from $U_{1}$. Continuing this way, one will eventually arrive at a $k$-isotropic subspace $V$ which is complementary to $W$. The first part of the proof then tells us that $V$ is in fact $k$-Lagrangian.

Notice that for $k+1=2$ we recover the well-known property from symplectic geometry which says that a Lagrangian subspace of a symplectic space always admits a Lagrangian complement. We now arrive at the main result of this section.

THEOREM 4.3. Let $(\mathscr{V}, \omega)$ be a multisymplectic vector space of order $k+1$. Then, $(\mathscr{V}, \omega)$ is isomorphic to a canonical multisymplectic space $\left(\mathscr{V}_{V}^{k}, \Omega\right)$ if and only if there exists a subspace $W$ of $\mathscr{V}$ such that: (i) $W$ is 1-Lagrangian, (ii) $\operatorname{dim} W=$ $\operatorname{dim} \bigwedge^{k}(\mathscr{V} / W)^{*}$ 
PROOF. The proof of the 'only if' part immediately follows from the considerations at the beginning of this section. Let us now assume that the conditions of the theorem hold for some subspace $W$ of $\mathscr{V}$. According to the previous proposition, there exists a $k$-Lagrangian subspace $V$ which is transversal to $W$, that is such that $\mathscr{V}=V \oplus W$. Consider then the following mapping:

$$
\phi: W \longrightarrow \bigwedge^{k} V^{*}, w \longmapsto-\left.\frac{1}{k+1}\left(i_{w} \omega\right)\right|_{V}
$$

Taking into account the assumptions (i) and (ii), one readily verifies that $\phi$ is a linear isomorphism. Next, we define the mapping

$$
\Psi: \mathscr{V}=V \oplus W \longrightarrow V \times \bigwedge^{k} V^{*}, v+w \longmapsto(v, \phi(w)),
$$

for any $v \in V$ and $w \in W$. It is easy to see that $\Psi$ is a linear isomorphism and, using (7) and the defining relation (3) for $\Omega$, it is straightforward to check that $\Psi^{*} \Omega=\omega$.

From this theorem we immediately deduce the following interesting property.

COROLLARY 4.4. Let $v$ be a volume form on a $(k+1)$-dimensional vector space $\mathscr{V}$, then the multisymplectic space $(\mathscr{V}, \nu)$ is isomorphic to a canonical multisymplectic space of order $k+1$.

PROOF. Take an arbitrary 1-dimensional subspace $W$ of $\mathscr{V}$. From Proposition 3.4 (iv) it follows that $W$ is 1-Lagrangian. Moreover, $p(=\operatorname{dim} W)=1$ verifies relation (6) and, hence, the conditions of the previous theorem are verified.

More explicitly, consider an arbitrary subspace $V$ of $\mathscr{V}$ transversal to $W$. Then, $\operatorname{dim} V=k$ and, again by Proposition 3.4 (iv), we know that $V$ is $k$-Lagrangian. Let $w$ be a non-zero vector in $W$ and put $\bar{v}=\left.i_{w} \nu\right|_{v}$. The $k$-form $\bar{v}$ is a volume form on $V$. The multisymplectic space $(\mathscr{V}, \nu)$ then is isomorphic to $\left(\mathscr{V}_{V}^{k}, \Omega\right)$, where $\Omega$ is given by

$$
\Omega\left(\left(v_{1}, \alpha_{1}\right), \ldots,\left(v_{k+1}, \alpha_{k+1}\right)\right)=\sum_{i=1}^{k+1}(-1)^{i} \lambda_{i} \bar{v}\left(v_{1}, \ldots, \hat{v}_{i}, \ldots, v_{k+1}\right),
$$

with $v_{i} \in V$ and $\alpha_{i}=\lambda_{i} \bar{\nu} \in \bigwedge^{k} V^{*}$ (see the Remark in Section 2).

As a further illustration of Theorem 4.3 , let us consider again the example $\left(\mathscr{V}_{0}, \omega_{0}\right)$ treated before. We have seen already that $W=\operatorname{span}\left\{e_{1}, e_{2}, e_{3}\right\}$ is a 1-Lagrangian subspace of $\mathscr{V}_{0}$. Moreover, $\operatorname{dim} W=3=\operatorname{dim} \bigwedge^{2}\left(\mathscr{V}_{0} / W\right)^{*}$. Consequently, $\left(\mathscr{V}_{0}, \omega_{0}\right)$ is isomorphic to a canonical multisymplectic space of order 3. A 3-Lagrangian subspace $V$ which is complementary to $W$ is given by $V=\operatorname{span}\left\{e_{3}-e_{4}, e_{5}, e_{6}\right\}$.

A simple example of a multisymplectic vector space which is not isomorphic to a canonical one, is obtained by considering a 5-dimensional vector space with basis $\left\{e_{i} \mid i=1, \ldots, 5\right\}$ and dual basis $\left\{\theta^{i} \mid i=1, \ldots, 5\right\}$. The 3-form $\omega=$ 
$\theta^{1} \wedge \theta^{2} \wedge \theta^{3}+\theta^{2} \wedge \theta^{3} \wedge \theta^{4}+\theta^{3} \wedge \theta^{4} \wedge \theta^{5}$ is nondegenerate, that is multisymplectic. However, it is readily seen that there exists no positive integer $p$ satisfying (6) with $n=5$ and $k=2$ and, therefore, a 5-dimensional multisymplectic space of order 3 can never be isomorphic to a canonical multisymplectic space.

In the next proposition, we identify some particular $k$-coisotropic and $k$-isotropic subspaces of $\left(\mathscr{V}_{V}^{k}, \Omega\right)$.

PROPOSITION 4.5. Let $U$ be a subspace of $V$ and let $U^{0} \subset V^{*}$ denote its annihilator.

(i) $U$, identified with $U \times\{0\}$, is a $k$-isotropic subspace of $\mathscr{V}_{V}^{k}$. In particular, we have

$$
U^{\perp, k}=V \times\left(\bigwedge^{k} V^{*} \cap \mathscr{I}\left(U^{0}\right)\right),
$$

where $\mathscr{I}\left(U^{0}\right)$ is the ideal in $\wedge V^{*}$ generated by $U^{0}$.

(ii) If $\operatorname{dim} U \geq k$, then $U \times \wedge^{k} V^{*}$ is a $k$-coisotropic subspace of $\left(\mathscr{V}_{V}^{k}, \Omega\right)$. In particular, we have

$$
\left(U \times \bigwedge^{k} V^{*}\right)^{\perp, k}=\left\{(0, \alpha) \mid \alpha \in \bigwedge^{k} V^{*} \cap \mathscr{I}\left(U^{0}\right)\right\} .
$$

Proof. (i) By definition, $(v, \alpha)$, with $v \in V$ and $\alpha \in \bigwedge^{k} V^{*}$, belongs to $U^{\perp, k}$ if and only if for all $u_{1}, \ldots, u_{k} \in U$

$$
0=\Omega\left((v, \alpha),\left(u_{1}, 0\right), \ldots,\left(u_{k}, 0\right)\right)=-\alpha\left(u_{1}, \ldots, u_{k}\right) .
$$

This holds if and only if $\alpha \in \mathscr{I}\left(U^{0}\right)$, which proves (8). Since, obviously, $U \times\{0\} \subset$ $V \times\left(\bigwedge^{k} V^{*} \cap \mathscr{I}\left(U^{0}\right)\right)$, it follows that, with the appropriate identification, $U$ is a $k$-isotropic subspace of $\mathscr{V}_{V}^{k}$.

(ii) Let $\left(e_{1}, \ldots, e_{m}\right)$ be a basis of $U$, with $m=\operatorname{dim} U \geq k$. We further complete this to a basis $\left(e_{1}, \ldots, e_{m}, e_{m+1}, \ldots, e_{n}\right)$ of $V$, and denote the dual basis by $\left(\theta^{1}, \ldots, \theta^{n}\right)$. Now, $(v, \alpha) \in\left(U \times \bigwedge^{k} V^{*}\right)^{\perp, k}$ if and only if $\Omega\left((v, \alpha),\left(u_{1}, \alpha_{1}\right), \ldots,\left(u_{k}, \alpha_{k}\right)\right)=0$, for all $u_{i} \in U$ and $\alpha_{i} \in \bigwedge^{k} V^{*}$. Putting, for instance, $u_{1}=0$, it follows from the definition (3) of $\Omega$ that

$$
\alpha_{1}\left(v, u_{2}, \ldots, u_{k}\right)=0
$$

for all $\alpha_{1} \in \bigwedge^{k} V^{*}$ and all $u_{i} \in U$. In particular,

$$
\left(\theta^{j} \wedge \theta^{i_{1}} \wedge \cdots \wedge \theta^{i_{k-1}}\right)\left(v, e_{i_{1}}, \ldots, e_{i_{k-1}}\right)=0
$$

for all $j>k, 1 \leq i_{1}<\cdots<i_{k-1} \leq k$. This clearly implies that $v \in U$. Relation (10) now reads $i_{v}\left(\left.\alpha_{1}\right|_{U}\right)=0$, for all $\alpha_{1} \in \bigwedge^{k} V^{*}$ and, hence, we necessarily have $v=0$. 
Next, again using (3) and the definition of $k$-th orthogonal complement, we see that $(0, \alpha) \in\left(U \times \wedge^{k} V^{*}\right)^{\perp, k}$ if and only if $\alpha\left(u_{1}, \ldots, u_{k}\right)=0$ for all $u_{i} \in U$. This holds if and only if $\alpha \in \mathscr{I}\left(U^{0}\right)$, which proves (9), and it then readily follows from this that $U \times \bigwedge^{k} V^{*}$ is $k$-coisotropic.

To close our discussion of the linear case, we wish to make a brief comparison between our notion of multisymplectic structure and the one studied by Martin [22, 23]. A multisymplectic vector space, as defined by Martin, corresponds to a multisymplectic space (in our sense) verifying the assumptions of Theorem 4.3 , with the additional restriction that codim $W>k$. Volume forms and structures of the kind described in Proposition 2.3, do not fit into this definition. We also note that in Martin's terminology, a Lagrangian subspace of a multisymplectic space of order $k+1$ corresponds to what we call a $k$-Lagrangian subspace (that is a Lagrangian subspace of 'highest order'). In [22] Martin has demonstrated that a multisymplectic space $(\mathscr{V}, \omega)$ of order $k+1$ (in his sense) always admits a special basis, called a Darboux basis, with respect to which the multisymplectic form $\omega$ takes on a 'canonical' form. More precisely, putting $\operatorname{dim} \mathscr{V}=n$ and $\operatorname{dim} W=p$, with $(n, p, k)$ satisfying (6), he has proved that there always exists a basis $\left\{f_{\left(i_{1}, \ldots i_{k}\right)} \mid 1 \leq i_{1}<\cdots<i_{k} \leq n-p\right\}$ of $W$, which can be extended to a basis $\left\{f_{\left(i_{1} \ldots i_{k}\right)}, e_{1}, \ldots, e_{n-p}\right\}$ of $\mathscr{V}$, such that in terms of the dual basis $\left\{\eta^{\left(i_{1} \ldots i_{k}\right)}, \theta^{1}, \ldots, \theta^{n-p}\right\}$, the multisymplectic form $\omega$ reads

$$
\omega=\sum_{1 \leq i_{1}<\cdots<i_{k} \leq n-p} \eta^{\left(i_{1} \ldots i_{k}\right)} \wedge \theta^{i_{1}} \wedge \ldots \wedge \theta^{i_{k}} .
$$

In some sense, Theorem 4.3 can be seen as providing an alternative proof for the existence of a Darboux basis for the class of multisymplectic spaces under consideration. Indeed, with respect to a natural basis for $V \times \wedge^{k} V^{*}$, the canonical multisymplectic form $\Omega$ on $\mathscr{V}_{v}^{k}$ will precisely be of the form (11). Note that one can also deduce from this that the 'length' of $\Omega$ equals the dimension of $\bigwedge^{k} V^{*}$ (see Remark 1 following Proposition 2.2).

\section{Multisymplectic manifolds}

Having defined the notion of multisymplectic vector space, one can subsequently introduce the notion of multisymplectic vector bundle as a vector bundle, the fibres of which possess a multisymplectic structure (of fixed order) with smooth dependence on the point in the base manifold. In case the vector bundle is the tangent bundle $T M$ of a smooth manifold $M$, a multisymplectic structure of order $k+1$ on $T M$ is defined by a differential $(k+1)$-form $\omega$ on $M$ which is nondegenerate in the sense that for a vector field $X$ on $M$

$$
i_{X} \omega=0 \text { if and only if } X=0 .
$$


The pair $(M, \omega)$ will then be called an almost multisymplectic manifold. Of special interest to us is the subclass of almost multisymplectic manifolds for which the almost multisymplectic structure is 'integrable' in the sense that the corresponding differential form $\omega$ is closed.

DEFINITION 5.1. A multisymplectic manifold $(M, \omega)$ of order $k+1$ consists of a smooth manifold $M$ and a closed $(k+1)$-form $\omega$ on $M$ which is nondegenerate (in the above sense). The differential form $\omega$ is called a multisymplectic form (of degree $k+1)$.

A submanifold $N$ of a multisymplectic manifold $(M, \omega)$ of order $k+1$ will be called l-isotropic (respectively $l$-coisotropic, l-Lagrangian), for $1 \leq l \leq k$, if at each point $n \in N, T_{n} N$ is a $l$-isotropic (respectively $l$-coisotropic, $l$-Lagrangian) subspace of the multisymplectic vector space $\left(T_{n} M, \omega_{n}\right)$. Using the obvious notation $T N^{\perp, l}=\bigcup_{n \in N}\left(T_{n} N\right)^{\perp, l}$, one can equivalently state that $N$ is l-isotropic (respectively $l$-coisotropic, $l$-Lagrangian) if $T N \subset T N^{\perp, l}$ (respectively $T N^{\perp, l} \subset T N, T N^{\perp, l}=$ $T N$ ). In the sequel we will only be concerned with submanifolds which are either 1or $k$-isotropic (respectively coisotropic, Lagrangian).

The results stated in the following proposition are straightforward extensions of the corresponding properties in symplectic geometry: see [1, Proposition 5.3.22 and Theorem 5.3.23], to which we also refer for the general idea of the proof.

PROPOSITION 5.2. $\quad$ (i) Let $N$ be a submanifold of a multisymplectic manifold $(M, \omega)$ of order $k+1$. If $T N \cap T N^{\perp, k}$ is a subbundle of $\left.T M\right|_{N}$, then it defines an integrable distribution on $N$ (and we denote the induced foliation by $\mathscr{N}$ ).

(ii) If, under the previous conditions, the quotient space $N / \mathscr{N}$ is a smooth manifold and the projection $\pi: N \rightarrow N / \mathscr{N}$ is a surjective submersion, then $N / \mathscr{N}$ admits a multisymplectic structure $\tilde{\omega}$ of order $k+1$ which is uniquely determined by $\pi^{*} \tilde{\omega}=i^{*} \omega$, where $i: N \hookrightarrow M$ denotes the inclusion map.

Given a closed $(k+1)$-form $\omega$ of constant rank on a manifold $M$, and assuming its characteristic distribution induces a regular foliation $\mathscr{C}$ on $M$, a standard argument shows that $\omega$ projects onto a multisymplectic form of degree $k+1$ on the quotient space $M / \mathscr{C}$. In such a case we may call $\omega$ pre-multisymplectic.

Symplectic manifolds and orientable manifolds (equipped with a volume form), are examples of multisymplectic manifolds. It is possible to show that, unlike symplectic structures, multisymplectic structures of higher order are much more abundant. In particular, for $3 \leq k+1 \leq n-3$, the set of multisymplectic structures is residual in the space of smooth $(k+1)$-forms on a $n$-dimensional manifold $M$, see [24, Theorem II 2.2 and Proposition II 4.2]. 
In the next section we will describe a canonical model of a multisymplectic manifold, which arises as a natural extension of the concept of a cotangent bundle. To conclude the present section we present some other interesting examples of manifolds which carry a multisymplectic structure.

EXAMPLES. (1) Let $(M, \Phi, \eta)$ be a cosymplectic manifold, that is $M$ is a $(2 n+1)$ dimensional smooth manifold with a closed 2-form $\Phi$ and a closed 1-form $\eta$, such that $\Phi^{n} \wedge \eta \neq 0$. In appropriate Darboux coordinates $\left(t, q^{i}, p_{i}\right)$ we have

$$
\eta=d t, \quad \Phi=d p_{i} \wedge d q^{i},
$$

and it is easily seen that the 3-form $\Omega=\eta \wedge \Phi$ is multisymplectic.

(2) Consider a compact semisimple Lie group $G$ and let $\langle$, $\rangle$ denote the nondegenerate left invariant metric on $G$, induced by the Killing form of its Lie algebra. One can then define the following 3-form on $G$ :

$$
\Omega(X, Y, Z)=\langle X,[Y, Z]\rangle+\langle Y,[Z, X]\rangle+\langle Z,[X, Y]\rangle
$$

for arbitrary vector fields $X, Y, Z$. It is routine to check that this form is closed and nondegenerate and, hence, $(G, \Omega)$ is a multisymplectic manifold of order 3 .

(3) For the definitions and properties related to the following example, we mainly rely on [31]. A $4 n$-dimensional manifold $M$ is called almost quaternionic if there is a subbundle $\mathscr{Q}$ of $\operatorname{End}(T M)$ such that for each $x \in M$ there exists a neighborhood $U$ of $x$ over which $\left.\mathscr{Q}\right|_{U}$ has a basis $\left\{J_{1}, J_{2}, J_{3}\right\}$ satisfying

$$
J_{1}^{2}=J_{2}^{2}=-1 \text { and } J_{3}=J_{1} J_{2}=-J_{2} J_{1} .
$$

In particular, the $J_{i}$ are three almost complex structures on $U$. A Riemannian metric $g$ on $M$ is said to be compatible with $\mathscr{Q}$ if at each point $x, g(A v, A w)=g(v, w)$ for all $v, w \in T_{x} M$ and $A \in \mathscr{Q}_{x}$ with $A^{2}=-1$. It can be shown that, given any Riemannian metric $g^{\prime}$ on an almost quaternionic manifold, one can always construct a compatible metric $g$. A triple $(M, \mathscr{Q}, g)$ consisting of an almost quaternionic manifold $(M, \mathscr{Q})$ with a compatible metric $g$, is called a quaternion-Hermitian manifold.

On a quaternion-Hermitian manifold $(M, \mathscr{Q}, g)$, the subbundle $\mathscr{Q}$ of End $(T M)$ can always be isometrically embedded into $\bigwedge^{2} T^{*} M$ by assigning to each element $A$ of $\mathscr{Q}$ the 2 -form $\omega_{A}$ defined by

$$
\omega_{A}(X, Y)=g(X, A Y) .
$$

This enables us to define, in terms of the above introduced local basis $\left\{J_{1}, J_{2}, J_{3}\right\}$ of $\mathscr{Q}$, the following 4-form on $M$

$$
\Omega=\omega_{J_{1}} \wedge \omega_{J_{1}}+\omega_{J_{2}} \wedge \omega_{J_{2}}+\omega_{J_{3}} \wedge \omega_{J_{3}}
$$


It can be shown that this form, which is called the fundamental 4-form, is globally defined and nondegenerate ([5,31] and references therein). A $4 n$-dimensional quaternion-Hermitian manifold $(M, \mathscr{Q}, g)$, with $n>1$, is said to be quaternionic almost Kähler if $d \Omega=0$, and quaternionic Kähler if $\nabla \Omega=0$, where $\nabla$ is the LeviCivita connection corresponding to $g$. Note that in the 4-dimensional case, $\Omega$ is a volume form and both $d \Omega$ and $\nabla \Omega$ then trivially vanish. A 4-dimensional quaternionHermitian manifold will be called quaternionic Kähler if it is Einstein and self-dual. Since $\nabla \Omega=0$ implies $d \Omega=0$, it follows that quaternionic Kähler manifolds are automatically quaternionic almost Kähler. Swann has proved that for $4 n \geq 12$ the converse also holds, that is $d \Omega=0$ implies $\nabla \Omega=0$ (see [31]).

From the above we may now conclude that a quaternionic (almost) Kähler manifold is multisymplectic, with multisymplectic form given by the fundamental 4 -form $\Omega$. An important special case is provided by the hyper-Kähler manifolds. A quaternionic Kähler manifold is called hyper-Kähler if the almost complex structures $J_{1}, J_{2}, J_{3}$ are globally defined and are such that $d \omega_{J_{i}}=0(i=1,2,3)$. Hitchin [14] has shown that this implies that $J_{1}, J_{2}, J_{3}$ are integrable and, hence, they define three Kähler structures on $M$.

\section{The bundle of exterior $k$-forms on a manifold}

As in the linear case, there is a straightforward way of constructing a class of manifolds which admit a canonical multisymplectic structure. Given an arbitrary smooth $n$-dimensional manifold $E$, let $\bigwedge^{k} E$ denote the bundle of exterior $k$-forms on $E$ (for $1 \leq k \leq n$ ), with canonical projection $\rho_{k}: \bigwedge^{k} E \rightarrow E$. This is a vector bundle with $C_{n}^{k}$-dimensional fibres: for $x \in E, \rho_{k}^{-1}(x)=\bigwedge^{k} T_{x}^{*} E$. For $k=1$ we have $\bigwedge^{1} E=T^{*} E$. On $\bigwedge^{k} E$ there exists a canonical $k$-form $\Theta_{E}$ which can be defined as follows. For $\alpha \in \bigwedge^{k} E$ and $v_{i} \in T_{\alpha}\left(\bigwedge^{k} E\right),(i=1, \ldots, k)$ put

$$
\left(\Theta_{E}\right)_{\alpha}\left(v_{1}, \ldots, v_{k}\right)=\alpha\left(\left(\rho_{k}\right)_{*}\left(v_{1}\right), \ldots,\left(\rho_{k}\right)_{*}\left(v_{k}\right)\right) .
$$

This is an immediate extension of the construction of the canonical Liouville 1-form on a cotangent bundle, which is also illustrated by the property that for any smooth $k$-form $\beta$ on $E$ we have

$$
\beta^{*}\left(\Theta_{E}\right)=\beta,
$$

where on the left-hand side $\beta$ is regarded as a section of $\rho_{k}$. Next, it is easy to verify that the closed $(k+1)$-form

$$
\Omega_{E}=d \Theta_{E}
$$


is nondegenerate and, hence, $\left(\bigwedge^{k} E, \Omega_{E}\right)$ is a multisymplectic manifold of order $k+1$.

Let $(U, \phi)$ be a local coordinate chart on $E$, with coordinates $\left(q^{1}, \ldots, q^{n}\right)$. On the corresponding chart $\left(\bigwedge^{k} U, \phi_{k}\right)$ of $\bigwedge^{k} E$ the coordinates are denoted by $\left(q^{i}, p_{i_{1} \ldots i_{k}}\right)$, with $1 \leq i_{1}<\cdots<i_{k} \leq n$. In terms of these bundle coordinates, the canonical forms $\Theta_{E}$ and $\Omega_{E}$ read:

$$
\Theta_{E}=p_{i_{1} \ldots i_{k}} d q^{i_{1}} \wedge \ldots \wedge d q^{i_{k}}, \quad \Omega_{E}=d p_{i_{1} \ldots i_{k}} \wedge d q^{i_{1}} \wedge \ldots \wedge d q^{i_{k}},
$$

with summation running over all $1 \leq i_{1}<\cdots<i_{k} \leq n$. The local model for the multisymplectic manifold $\left(\bigwedge^{k} E, \Omega_{E}\right)$ is the vector space $\mathscr{V}_{\mathbb{R}^{n}}^{k}=\mathbb{R}^{n} \times \bigwedge^{k} \mathbb{R}^{n}$, equipped with its canonical multisymplectic structure. Indeed, if $\phi(U)=U^{\prime}$ (an open subset of $\left.\mathbb{R}^{n}\right)$, then $\phi_{*}\left(\bigwedge^{m} U\right)=U^{\prime} \times \bigwedge^{k} \mathbb{R}^{n}$ and, clearly, $\left.\Omega_{E}\right|_{U}$ is $\phi_{*}$-related to the canonical multisymplectic form of $\mathscr{V}_{\mathbb{R}^{n}}^{k}$ which is precisely of the form (11).

As on any vector bundle, there exists a canonical dilation vector field on $\bigwedge^{k} E$, which is here given by

$$
\Delta_{E}=\sum_{1 \leq i_{1}<\ldots<i_{k} \leq n} p_{i_{1} \ldots i_{k}} \frac{\partial}{\partial p_{i_{1} \ldots i_{k}}} .
$$

Using (13) it is straightforward to check that $\Delta_{E}$ is also uniquely determined by

$$
i_{\Delta_{E}} \Omega_{E}=\Theta_{E}
$$

We now consider the interesting case where $E$ itself is fibred over some manifold $M$, with projection $\pi: E \rightarrow M$. For any $r$, with $0 \leq r \leq k-1$, let $\wedge_{r}^{k} E$ denote the bundle over $E$ consisting of those exterior $k$-forms on $E$ which vanish whenever $r+1$ of its arguments are vertical tangent vectors (with respect to the given fibration). Clearly, $\bigwedge_{r}^{k} E$ is a subbundle of $\bigwedge^{k} E$ and smooth sections of the bundle $\bigwedge_{0}^{k} E$ are semi-basic $k$-forms on $E$ with respect to $\pi$. Let $i_{k, r}: \bigwedge_{r}^{k} E \hookrightarrow \bigwedge^{k} E$ denote the natural inclusion. Using Proposition 2.3 it is then straightforward to prove the following result.

PROPOSITION 6.1. The $(k+1)$-form $i_{k, r}{ }^{*}\left(\Omega_{E}\right)$ is a multisymplectic form on $\bigwedge_{r}^{k} E$.

In particular, for $r=1$ this precisely yields the kind of multisymplectic structure appearing in first order field theories [6, 10,33].

One of the characteristic features of a cotangent bundle $T^{*} E$, with its canonical symplectic structure, is the existence of a polarization which admits a transverse Lagrangian section (namely, the Lagrangian foliation determined by the fibres and the zero section, identified with the base manifold $E$ ). A similar property now holds for the canonical multisymplectic manifolds $\bigwedge^{k} E$, except that for $k>1$, the fibres and the zero section are Lagrangian submanifolds of a 'different order'. More generally, 
in the next proposition we prove that the image of any closed $k$-form on $E$ (regarded as a section of $\rho_{k}$ ) determines a $k$-Lagrangian submanifold of $\bigwedge^{k} E$, whereas the fibres are 1-Lagrangian submanifolds. Herewith, we have identified on $\left(\bigwedge^{k} E, \Omega_{E}\right)$ the analogue of the two canonical types of Lagrangian submanifolds encountered in the symplectic geometry of a cotangent bundle.

PROPOSITION 6.2. The fibres of $\rho_{k}: \bigwedge^{k} E \rightarrow E$ are 1-Lagrangian submanifolds of $\left(\bigwedge^{k} E, \Omega_{E}\right.$ ) and the graph of any closed $k$-form on $E$ (and thus also, in particular, the zero section of $\rho_{k}$ ) is a $k$-Lagrangian submanifold.

Proof. The local model for $\left(\bigwedge^{k} E, \Omega_{E}\right)$ tells us that at each point $\alpha \in \bigwedge^{k} E$ we have

$$
T_{\alpha}\left(\bigwedge^{k} E\right) \cong \mathbb{R}^{n} \times \bigwedge^{k} \mathbb{R}^{n}
$$

with $n=\operatorname{dim} E$. On the other hand, for the tangent space to the fibre through $\alpha$ we find

$$
T_{\alpha}\left(\rho_{k}{ }^{-1}\left(\rho_{k}(\alpha)\right)\right) \cong \bigwedge^{k} \mathbb{R}^{n}
$$

and, hence, it follows from Theorem 4.1 that this is a 1-Lagrangian subspace of $T_{\alpha}\left(\bigwedge^{k} E\right)$.

Next, let $\beta$ be an arbitrary closed $k$-form on $E$. It follows from the considerations at the beginning of this section that

$$
\beta^{*} \Omega_{E}=d\left(\beta^{*} \Theta_{E}\right)=d \beta=0 .
$$

This already implies that $\beta(E)$ is a $k$-isotropic submanifold of $\bigwedge^{k} E$. Consequently, at each point $\alpha \in \beta(E), T_{\alpha} \beta(E)$ is a $k$-isotropic subspace of $T_{\alpha}\left(\bigwedge^{k} E\right)$ which, moreover, is transversal to the 1-Lagrangian subspace $T_{\alpha}\left(\rho_{k}^{-1}\left(\rho_{k}(\alpha)\right)\right)$. The argument used at the beginning of the proof of Proposition 4.2 then shows that $T_{\alpha} \beta(E)$ is in fact $k$-Lagrangian.

In the remainder of this section we will put, for notational convenience, $E^{(k)}=$ $\bigwedge^{k} E$. The subbundle of $T E^{(k)}$ consisting of all tangent vectors that are vertical with respect to $\rho_{k}$, is denoted by $V E^{(k)}$. We will show that there exists a canonical vertical lift operation mapping any $k$-form on $E$ into a vertical vector field on $E^{(k)}$. First, we establish an isomorphism between $V E^{(k)}$ and $\bigwedge_{0}^{k} E^{(k)}$, the bundle of exterior $k$-forms on $E^{(k)}$ that are horizontal (or basic) with respect to the projection onto $E$.

LEMMA 6.3. The mapping $\Phi: T E^{(k)} \rightarrow \bigwedge^{k} E^{(k)}, v \in T_{\alpha} E^{(k)} \mapsto i_{v} \Omega_{E}(\alpha)$ induces a vector bundle isomorphism between $V E^{(k)}$ and $\bigwedge_{0}^{k} E^{(k)}$. 
PROOF. Since $\Omega_{E}$ is nondegenerate, $\Phi$ is necessarily injective. The previous proposition tells us that the fibers of $E^{(k)}$ are 1-Lagrangian. From this one can easily deduce that

$$
\Phi\left(V E^{(k)}\right) \subset \bigwedge_{0}^{k} E^{(k)}
$$

Since, obviously, $\Phi$ is linear, the result now immediately follows by observing that $\operatorname{dim} V E^{(k)}=\operatorname{dim} \bigwedge_{0}^{k} E^{(k)}$.

Herewith we now derive the following lifting procedure of $k$-forms on $E$ to vertical vector fields on $E^{(k)}$.

PROPOSITION 6.4. There exists a mapping which sends any $k$-form $\beta$ on $E$ into a vertical vector field $\beta^{v}$ on $E^{(k)}\left(=\bigwedge^{k} E\right)$, where $\beta^{v}$ is uniquely determined by

$$
i_{\beta^{v}} \Omega_{E}=\rho_{k}^{*} \beta
$$

Moreover, for any two k-forms $\beta$ and $\gamma$ on $E$ we have:

$$
\left[\beta^{v}, \gamma^{v}\right]=0
$$

PROOF. The fact that (14) uniquely determines a vertical vector field $\beta^{v}$, for given $\beta$, is an immediate consequence of the previous lemma.

In order to prove (15), note that

$$
\begin{aligned}
i_{\left[\beta^{v}, \gamma^{v}\right]} \Omega_{E} & =\mathscr{L}_{\beta^{v}}\left(i_{\gamma^{v}} \Omega_{E}\right)-i_{\gamma^{v}}\left(\mathscr{L}_{\beta^{v}} \Omega_{E}\right) \\
& =\mathscr{L}_{\beta^{v}}\left(\rho_{k}^{*} \gamma\right)-i_{\gamma^{v}} d\left(i_{\beta^{v}} \Omega_{E}\right) \\
& =i_{\beta^{v}} d\left(\rho_{k}^{*} \gamma\right)+d i_{\beta^{v}} \rho_{k}^{*} \gamma-i_{\gamma^{v}} d\left(\rho_{k}^{*} \beta\right),
\end{aligned}
$$

where the symbol $\mathscr{L}$ denotes the Lie derivative operator. Now, $d\left(\rho_{k}^{*} \beta\right), \rho_{k}^{*} \gamma$ and $d\left(\rho_{k}^{*} \gamma\right)$ are basic forms on $E^{(k)}$. Hence, $\beta^{v}$ and $\gamma^{v}$ being vertical vector fields on $E^{(k)}$, it follows that

$$
i_{\left[\beta^{v}, \mathcal{\gamma}^{v}\right]} \Omega_{E}=0,
$$

and therefore, since $\Omega_{E}$ is nondegenerate, $\left[\beta^{v}, \gamma^{v}\right]=0$.

Note again that the previous results are immediate extensions of known results for cotangent bundles (see [37]). 


\section{Polarized multisymplectic manifolds}

Proposition 6.2 tells us that the canonical multisymplectic manifold $\left(\bigwedge^{k} E, \Omega_{E}\right)$ possesses a 1-Lagrangian foliation with a transversal $k$-Lagrangian section. Moreover, the leaves of this foliation all have the same dimension, namely

$$
\operatorname{dim} \rho_{k}^{-1}(x)=\operatorname{dim} \bigwedge^{k} T_{x}^{*} E .
$$

These observations prompt us to introduce the following definition.

DEFINITION 7.1. A polarized multisymplectic manifold is a triple $(M, \omega, \mathscr{F})$ consisting of a multisymplectic manifold $(M, \omega)$ and a foliation $\mathscr{F}$, the leaves of which are immersed 1-Lagrangian submanifolds which all have the same dimension.

Note that, unlike in the symplectic case, 1-Lagrangian submanifolds (and, more generally, $l$-Lagrangian submanifolds) of a given multisymplectic manifold need not have a fixed dimension (see, in that respect, the comment made at the end of Section 3). Therefore, it may happen that a multisymplectic manifold of order $k+1>2$ admits a 'generalized' foliation with 1-Lagrangian leaves which do not all have the same dimension.

REMARK. The notion of multisymplectic manifold considered by Martin [22] corresponds, in our terminology, to a $n$-dimensional polarized multisymplectic manifold $(M, \omega, \mathscr{F})$ of order $k+1$, where $\mathscr{F}$ is induced by an integrable 1-Lagrangian distribution of constant rank $p$, such that the triple $(n, p, k)$ satisfies the relation (6). Generalizing a result of Weinstein for symplectic manifolds with a Lagrangian foliation (see [34]), Martin then further proves the following. If there exists a global transversal $k$-Lagrangian section $L$ of $\mathscr{F}$, then there exists a tubular neighbourhood $U$ of $L$, a manifold $E$ and a multisymplectic diffeomorphism between $\left(U,\left.\omega\right|_{U}\right)$ and $\left(W,\left.\Omega_{E}\right|_{W}\right.$ ), with $W$ a neighbourhood of the zero section in $\wedge^{k} E$.

Given a polarized multisymplectic manifold $(M, \omega, \mathscr{F})$, the foliation $\mathscr{F}$ is called regular if the space of leaves $M / \mathscr{F}$ admits a smooth manifold structure such that $M$ becomes a fibred manifold over $M / \mathscr{F}$ with the canonical projection $\rho: M \rightarrow M / \mathscr{F}$ being a surjective submersion. Taking into account Proposition 3.4 (ii) it can then be easily deduced that $\operatorname{dim} M / \mathscr{F} \geq k$.

DEFINITION 7.2. A polarized multisymplectic manifold $(M, \omega, \mathscr{F})$ of order $k+1$ will be called saturated if $\mathscr{F}$ is a regular foliation and if, moreover, for each $x \in M / \mathscr{F}$ :

$$
\operatorname{dim} \rho^{-1}(x)=\operatorname{dim} \bigwedge^{k} T_{x}^{*}(M / \mathscr{F}),
$$


Clearly, $\wedge^{k} E$ with its canonical multisymplectic structure $\Omega_{E}$ is a saturated polarized multisymplectic manifold. In the remainder of this section we intend to analyse in more detail the structure of saturated polarized multisymplectic manifolds. In particular, we will establish conditions for such a manifold to become an affine bundle modelled on a bundle of exterior forms. We thereby proceed entirely along the same lines as in [32], where a similar result was presented for symplectic manifolds with a Lagrangian fibration.

First we observe that, in full analogy with the construction in Proposition 6.4, a saturated polarized multisymplectic manifold $(M, \omega, \mathscr{F})$ of order $k+1$ admits a vertical lift operation, assigning to each $k$-form $\beta$ on $M / \mathscr{F}$ a vertical vector field $\beta^{v}$ on $M$ according to the prescription

$$
i_{\beta^{v}} \omega=\rho^{*} \beta,
$$

and such that any two vertical lifts commute. For each $x \in M / \mathscr{F}$ and any $p \in \rho^{-1}(x)$, the vertical lift induces an isomorphism between the vector spaces $\bigwedge^{k} T_{x}^{*}(M / \mathscr{F})$ and $V_{p} M=T_{p}\left(\rho^{-1}(x)\right)$ :

$$
\begin{aligned}
\phi_{p}: \bigwedge^{k} T_{x}^{*}(M / \mathscr{F}) & \longrightarrow V_{p} M \\
\alpha & \longmapsto \phi_{p}(\alpha)=\tilde{\alpha}^{v}(p),
\end{aligned}
$$

where $\tilde{\alpha}$ is any (local) $k$-form on $M / \mathscr{F}$ for which $\tilde{\alpha}(x)=\alpha$. We will also use the notation $\alpha^{v}$ for the vector field on $\rho^{-1}(x)$ defined by $\alpha^{v}(p)=\phi_{p}(\alpha)$, that is $\alpha^{v}=\left.\tilde{\alpha}^{v}\right|_{\rho^{-1}(x)}$.

We can now state the following structure theorem for saturated polarized multisymplectic manifolds.

THEOREM 7.3. Let $(M, \omega, \mathscr{F})$ be a saturated polarized multisymplectic manifold of order $k+1$ and put $M / \mathscr{F}=E$. Let $\rho: M \rightarrow E$ denote the natural projection and assume all the fibres of $\rho$ are connected. Then:

(i) For each $x \in E$, the corresponding fibre $\rho^{-1}(x)$ is an affine manifold (that is, it admits a flat, torsion free, linear connection).

(ii) If, in addition, all the fibres of $\rho$ are simply connected and complete (with respect to its affine structure), then $M$ is an affine bundle modelled on $\wedge^{k} E$. Any section s of $\rho$ determines a diffeomorphism $\Phi_{s}: M \rightarrow \wedge^{k} E$ such that

$$
\Phi_{s}^{*} \Omega_{E}=\omega+\rho^{*} \omega_{s}
$$

for some closed $(k+1)$-form $\omega_{s}$ on $E$. Moreover, the cohomology class $\left[\omega_{s}\right] \in$ $H^{k+1}(E, \mathbb{R})$ is independent of the chosen section s. 
PROOF. The proof is completely similar to the one given by Thompson for the symplectic case (see [32, Theorem 2.3]), to which we refer for the details.

(i) From the above we know that for each $x \in E$ and $p \in \rho^{-1}(x)$, there exists an isomorphism $\phi_{p}$ between $\wedge^{k} T_{x}^{*} E$ and $T_{p}\left(\rho^{-1}(x)\right)$. Given a positive definite metric $\langle$,$\rangle on the linear space \bigwedge^{k} T_{x}^{*} E$, one can then define a Riemannian metric $g$ on the fiber $\rho^{-1}(x)$ according to

$$
g_{p}\left(v_{1}, v_{2}\right)=\left\langle\phi_{p}^{-1}\left(v_{1}\right), \phi_{p}^{-1}\left(v_{2}\right)\right\rangle,
$$

for all $p \in \rho^{-1}(x)$ and $v_{1}, v_{2} \in T_{p}\left(\rho^{-1}(x)\right)$. Note that for any $\alpha_{1}, \alpha_{2} \in \bigwedge^{k} T_{x}^{*} E$, we have $g\left(\alpha_{1}{ }^{v}, \alpha_{2}{ }^{v}\right)=\left\langle\alpha_{1}, \alpha_{2}\right\rangle$. It can then be shown that for each $\alpha \in \bigwedge^{k} T_{x}^{*} E$, the (vertical) vector field $\alpha^{v}$ on $\rho^{-1}(x)$ is a Killing vector field with constant length with respect to $g$. Now, starting from a basis $\left(\alpha_{i}\right)$ of $\wedge^{k} T_{x}^{*} E$, the corresponding vertical lifts $\left(\alpha_{i}{ }^{v}\right)$ determine a basis for the module of vector fields on the fibre $\rho^{-1}(x)$, consisting of pairwise commuting Killing vector fields with constant inner product relative to $g$. It then easily follows that the Levi-Civita connection corresponding to $g$ has zero curvature. Each fibre thus admits a flat, symmetric linear connection and, therefore, is an affine manifold.

(ii) For $\alpha \in \bigwedge^{k} T_{x}^{*} E$ and $p \in \rho^{-1}(x)$, let $\gamma_{\alpha}(p,):. \mathbb{R} \rightarrow \rho^{-1}(x)$ denote the unique integral curve of $\alpha^{v}$ for which $\gamma_{\alpha}(p, 0)=p$. Since vertical lifts commute, we find that for any $\alpha_{1}, \alpha_{2} \in \bigwedge^{k} T_{x}^{*} E, \gamma_{\alpha_{2}}\left(\gamma_{\alpha_{1}}(p, t), t\right)=\gamma_{\alpha_{2}+\alpha_{1}}(p, t)$. Again following the same argument as in [32] one can then prove that, under the given assumptions, the map

$$
\lambda_{x}: \bigwedge^{k} T_{x}^{*} E \times \rho^{-1}(x) \longrightarrow \rho^{-1}(x),(\alpha, p) \longmapsto \gamma_{\alpha}(p, 1),
$$

determines a free and transitive action of the vector space $\wedge^{k} T_{x}^{*} E$, regarded as an additive Lie group, on $\rho^{-1}(x)$. Hence, $\rho^{-1}(x)$ is an affine space modelled on $\wedge^{k} T_{x}^{*} E$. Since this holds for each fibre of $\rho$, it easily follows that $(M, \rho, E)$ is an affine bundle modelled on the vector bundle $\left(\bigwedge^{k} E, \rho_{k}, E\right)$.

Let $s: E \rightarrow M$ be an arbitrary global section of $\rho$. (Affine bundles, having contractible fibres, always admit global sections.) This induces a vector bundle structure on $M$ and we can then construct a bundle isomorphism $\Phi_{s}: M \rightarrow \wedge^{k} E$ as follows. For each $p \in M$, with $\rho(p)=x$, let $\Phi_{s}(p)$ be that element of $\wedge^{k} T_{x}^{*} E$ which is uniquely determined by $\lambda_{x}\left(\Phi_{s}(p), s(x)\right)=p$. In particular, we have $\rho_{k} \circ \Phi_{s}=\rho$ and $\Phi_{s}$ maps $s(E)$ onto the zero section of $\bigwedge^{k} E$. Consider then the $(k+1)$-form

$$
\tilde{\omega}_{s}=\Phi_{s}^{*} \Omega_{E}-\omega
$$

on $M$, which is obviously closed. Moreover, for any $k$-form $\beta$ on $E$ one easily finds that $i_{\beta^{v}} \tilde{\omega}_{s}=0$ and $\mathscr{L}_{\beta^{v}} \tilde{\omega}_{s}=0$. Since at each $p \in M$, the vertical tangent space $V_{p} M$ 
is spanned by the vertical lifts of $k$-forms, it follows from the previous considerations that $\tilde{\omega}_{s}$ is the pull-back of a $(k+1)$-form $\omega_{s}$ on $E$, that is

$$
\tilde{\omega}_{s}=\rho^{*} \omega_{s}
$$

and, $\rho$ being a surjective submersion, $\omega_{s}$ is necessarily closed. This completes the proof of (16). Finally, given any two sections $s_{1}, s_{2}$ of $\rho$ we have

$$
\rho^{*} \omega_{s_{1}}-\rho^{*} \omega_{s_{2}}=\Phi_{s_{1}}^{*} \Omega_{E}-\Phi_{s_{2}}^{*} \Omega_{E}=d\left(\Phi_{s_{1}}^{*} \Theta_{E}-\Phi_{s_{2}}^{*} \Theta_{E}\right),
$$

from which it follows that $\omega_{s_{1}}$ and $\omega_{s_{2}}$ belong to the same cohomology class.

The previous theorem tells us that each saturated polarized multisymplectic manifold $(M, \omega, \mathscr{F})$ of order $k+1$, satisfying the appropriate topological assumptions, determines a unique element of $H^{k+1}(M / \mathscr{F}, \mathbb{R})$.

We will say that a saturated polarized multisymplectic manifold $(M, \omega, \mathscr{F})$ of order $k+1$ is equivalent to $\left(\bigwedge^{k} E, \Omega_{E}\right.$ ), with $E=M / \mathscr{F}$, if $\omega=d \theta$ for some $k$-form $\theta$ on $M$ and if there exists a bundle isomorphism $\Phi: M \rightarrow \bigwedge^{k} E$ over the identity such that $\Phi^{*} \Theta_{E}=\theta$. From Theorem 7.3 one can now easily deduce the following result.

COROLLARY 7.4. A saturated polarized multisymplectic manifold $(M, \omega, \mathscr{F})$ of order $k+1$ which verifies all the assumptions of Theorem 7.3, is equivalent to $\left(\bigwedge^{k} E, \Omega_{E}\right)$ if and only if it determines the zero cohomology class in $H^{k+1}(E, \mathbb{R})$.

The proof is rather straightforward and is again completely similar to the one given for the symplectic case in [32, Corollary 2.4].

Finally, it is interesting to compare the above approach to the characterization of a bundle of exterior forms, with Nagano's approach to the characterization of a cotangent bundle structure (see [25]). More precisely, Nagano has established necessary and sufficient conditions for an exact symplectic manifold $(M, d \theta)$ to be a cotangent bundle such that $\theta$ is the canonical Liouville 1-form. A central role in his analysis is played by the canonical vector field $\Delta$ on $M$ which is determined by the relation $i_{\Delta} d \theta=\theta$. In particular, Nagano has given necessary and sufficient conditions for $\Delta$ to induce a vector bundle structure on $M$ with respect to which it becomes the dilation vector field. These conditions are the following:

(a) $\Delta$ is complete;

(b) for each $x \in M, \lim _{t \rightarrow-\infty} \exp (t \Delta)(x)$ determines a unique point in $M$;

(c) at each singular point $x_{0}$ of $\Delta$, the linearization $\Delta^{\prime}\left(x_{0}\right): T_{x_{0}} M \rightarrow T_{x_{0}} M$ is idempotent;

(d) the set of singular points $x_{0}$ of $\Delta$ constitute a submanifold of $M$ of codimension equal to the rank of $\Delta^{\prime}\left(x_{0}\right)$ (this rank being a constant, independent of $x_{0}$ ). 
We can now state the following generalization of Nagano's result, the proof being completely similar to the one given in [25, Theorem 4.1].

THEOREM 7.5. Let $(M, d \theta)$ be a multisymplectic manifold of order $k+1$ and suppose there exists a (necessarily unique) vector field $\Delta$ such that $i_{\Delta} d \theta=\theta$. Then, $M$ admits the structure of a bundle of exterior $k$-forms $\wedge^{k} E$ on a manifold $E$, such that $\theta$ becomes the canonical $k$-form $\Theta_{E}$, if and only if $\Delta$ verifies the above conditions (a), (b), (c) and (d). Moreover, this exterior bundle structure is unique in the sense that $E$ is determined up to a diffeomorphism.

Note that for an exact multisymplectic structure $(M, d \theta)$ of order $k+1>2$ it need not be true, in general, that there exists a vector field $\Delta$ on $M$ for which $i_{\Delta} d \theta=\theta$. This is due to the fact that on a (finite dimensional) multisymplectic manifold $(M, \omega)$ of order $k+1$, the 'musical map' $X \rightarrow i_{X} \omega$, which sends vectors into $k$-forms, is bijective in the symplectic case and in the case of a volume form, whereas it is merely injective in the general case (for $2<k+1<\operatorname{dim} M$ ). This explains why we have to include the existence of $\Delta$ as an additional assumption in the above theorem. A simple counterexample, however, is provided by the following. Consider the 2 -form $\theta$ on $\mathbb{R}^{5}$ given by $\theta=x^{1} d x^{2} \wedge d x^{3}+x^{2} d x^{3} \wedge d x^{4}+x^{3} d x^{4} \wedge d x^{5}$. One easily verifies that $d \theta=d x^{1} \wedge d x^{2} \wedge d x^{3}+d x^{2} \wedge d x^{3} \wedge d x^{4}+d x^{3} \wedge d x^{4} \wedge d x^{5}$ is nondegenerate, but there exists no vector field on $\mathbb{R}^{5}$ whose contraction with $d \theta$ equals $\theta$.

In forthcoming work we intend to investigate, among others, symmetry and reduction properties of multisymplectic structures, Poisson brackets induced by multisymplectic forms and generalized Hamiltonian systems on multisymplectic manifolds.

\section{Acknowledgements}

This research was partially supported by a NATO Collaborative Research Grant (No. CRG 940195). First author thanks the Fund for Scientific Research - Flanders (Belgium) for continuing support. Second author wishes to thank for partial financial support from the DGICYT project PB95-0401, and third author for support from the DGICYT project PB94-0106. Finally, the authors thank the referee for bringing the papers $[24,36]$ to their attention.

\section{References}

[1] R. Abraham and J. E. Marsden, Foundations of mechanics (Benjamin Cummings, Reading, MA, 1978).

[2] A. Awane, ' $k$-symplectic structures', J. Math. Phys. 33 (1992), 4046-4052. 
[3] ___ ' G-espaces $K$-symplectiques homogènes', J. Geom. Phys. 13 (1994), 139-157.

[4] E. Binz, J. Śniatycki and H. Fisher, Geometry of classical fields, Math. Studies 154 (North-Holland, Amsterdam, 1989).

[5] E. Bonan, 'Sur l'algèbre extérieure d'une variété hermitienne quaternionique', $C$. R. Acad. Sci. Paris (Série l) 295 (1982), 115-118.

[6] J. F. Cariñena, M. Crampin and L. A. Ibort, 'On the multisymplectic formalism for first order field theories', Differential Geom. Appl. 1 (1991), 345-374.

[7] R. O. Fulp, J. K. Lawson and L. K. Norris, 'Generalized symplectic geometry as a covering theory for Hamiltonian theories of classical particles and fields', J. Geom. Phys. 20 (1996), 195-206.

[8] P. L. Garcia and A. Perez-Rendon, 'Symplectic approach to the theory of quantized fields II', Arch. Rational Mech. Anal. 43 (1971), 101-124.

[9] H. Goldschmidt and S. Sternberg, 'The Hamilton-Cartan formalism in the calculus of variations', Ann. Inst. Fourier (Grenoble) 23 (1973), 203-267.

[10] M. J. Gotay, 'A multisymplectic framework for classical field theory and the calculus of variations. I. Covariant Hamiltonian formalism', in: Mechanics, analysis and geometry: 200 years after Lagrange (ed. M. Francaviglia) (Elsevier, New York, 1991) pp. 203-235.

[11] _ _ 'A multisymplectic framework for classical field theory and the calculus of variations. II. Space + time decomposition', Differential Geom. Appl. 1 (1991), 375-390.

[12] M. Gotay, J. Isenberg, J. E. Marsden and R. Montgomery, 'Momentum mappings and the Hamiltonian structure of classical field theories with constraints', Technical report.

[13] C. Günther, 'The polysymplectic Hamiltonian formalism in field theories and the calculus of variations', J. Differential Geom. 25 (1987), 23-53.

[14] N. J. Hitchin, 'The self-duality equations on a Riemann surface', Proc. London Math. Soc. 55 (1987), 59-126.

[15] A. Ibort, 'The geometry of moduli spaces of Yang-Mills theories in dimension 3 and 4', An. de Fisica, Monografias RSEF 3 (1995), 151-165.

[16] I. Kanatchikov, 'Novel algebraic structures from the polysymplectic form in field theory', in: GROUP 21, Physical applications and mathematical aspects of geometry, groups and algebras, vol. 2 (eds. H.-D. Doebner, W. Scherer and C. Schulte) (World Scientific, London, 1997) pp. 894-899.

[17] J. Kijowski and W. Szczyrba, 'Multisymplectic manifolds and the geometrical construction of the Poisson brackets in the classical field theory', in: Géométrie symplectique et physique mathématique, Coll. Int. C.N.R.S. No 237 (C.N.R.S., 1975) pp. 347-378.

[18] J. Kijowski, 'A finite-dimensional canonical formalism in the classical field theory', Commun. Math. Phys. 30 (1973), 99-128.

[19] J. Kijowski and W. M. Tulczyjew, A symplectic framework for field theories, Lecture Notes in Phys. 107 (Springer, Berlin, 1979).

[20] M. de León, I. Mendez and M. Salgado, 'p-almost cotangent structures', Boll. Un. Mat. Ital. A-7 (1993), 97-107.

[21] P. Libermann and C.-M. Marle, Symplectic geometry and analytical mechanics, Mathematics and its Applications (D. Reidel, Boston, 1987).

[22] G. Martin, 'A Darboux theorem for multi-symplectic manifolds', Lett. Math. Phys. 16 (1988), 133-138.

[23] _ 'Dynamical structures for $k$-vector fields', Internat. J. Theoret. Phys. 27 (1988), 571-585.

[24] J. Martinet, 'Sur les singularités des formes différentielles', Ann. Inst. Fourier (Grenoble) 20 (1970), 95-178.

[25] T. Nagano, '1-forms with the exterior derivative of maximal rank', J. Differential Geom. 2 (1968), 253-264. 
[26] L. K. Norris, 'Generalized symplectic geometry on the frame bundle of a manifold', Proc. Sympos. Pure Math. 54 (1993), 435-465.

[27] P. Revoy, 'Trivecteurs de rang 6', Bull. Soc. Math. France, Mem. 59 (1979), 141-155.

[28] G. Sardanashvily, Generalized Hamiltonian formalism for field theory. Constraint Systems (World Scientific, London, 1995).

[29] G. Sardanashvily and O. Zakharov, 'On application of the Hamiltonian formalism in fibred manifolds to field theory', Differential Geom. Appl. 3 (1993), 245-263.

[30] J. Śniatycki, 'On the geometric structure of classical field theory in Lagrangian formulation', Proc. Camb. Phil. Soc. 68 (1970), 475-484.

[31] A. Swann, 'Hyper-Kähler and quaternionic Kähler geometry', Math. Ann. 289 (1991), 421-450.

[32] G. Thompson, 'Symplectic manifolds with Lagrangian fibration', Lett. Math. Phys. 12 (1986), 241-248.

[33] W. M. Tulczyjew, 'Homogeneous symplectic formulation of field dynamics and the PoincaréCartan form', in: Differential geometrical methods in mathematical physics, Lecture Notes in Math. 836 (Springer, Berlin, 1980) pp. 484-497.

[34] A. Weinstein, 'Symplectic manifolds and their Lagrangian submanifolds', Adv. Math. 6 (1971), 329-346.

[35] R. Westwick, 'Irreducible lengths of trivectors of rank seven and eight', Pacific J. Math. 80 (1979), $575-579$.

[36] - 'Real trivectors of rank seven', Linear and Multilinear Algebra 10 (1981), 183-204.

[37] K. Yano and S. Ishihara, Tangent and cotangent bundles (Marcel Dekker, New York, 1973).

Theoretical Mechanics Division

University of Gent

Krijgslaan 281

B-9000 Gent

Belgium

Instituto de Matemáticas y Física Fundamental

Consejo Superior de Investigaciones Científicas

Serrano 123

E-28006 Madrid

Spain

\author{
Departamento de Matematicas \\ Universidad Carlos III de Madrid \\ E-28911 Leganes, Madrid \\ Spain
}

